\title{
Energy Loss of Hydrogen- and Helium-Ion Beams in DNA: Calculations Based on a Realistic Energy-Loss Function of the Target
}

\author{
Isabel Abril, ${ }^{a, 1}$ Rafael Garcia-Molina, ${ }^{b, 1,2}$ Cristian D. Denton, ${ }^{a}$ Ioanna Kyriakou ${ }^{c}$ and Dimitris Emfietzoglou ${ }^{c}$
}

a Departament de Física Aplicada, Universitat d'Alacant, E-03080 Alacant, Spain; ${ }^{b}$ Departamento de Física - Centro de Investigación en Optica y Nanofisica (CIOyN), Universidad de Murcia, E-30100 Murcia, Spain; and c Medical Physics Laboratory, University of Ioannina, Medical School, GR-45110 Ioannina, Greece

\begin{abstract}
Abril, I., Garcia-Molina, R., Denton, C. D., Kyriakou, I. and Emfietzoglou, D. Energy Loss of Hydrogen- and Helium-Ion Beams in DNA: Calculations Based on a Realistic Energy-Loss Function of the Target. Radiat. Res. 175, 247-255 (2011).
\end{abstract}

We have calculated the electronic energy loss of proton and $\alpha$ particle beams in dry DNA using the dielectric formalism. The electronic response of DNA is described by the MELF-GOS model, in which the outer electron excitations of the target are accounted for by a linear combination of Mermin-type energyloss functions that accurately matches the available experimental data for DNA obtained from optical measurements, whereas the inner-shell electron excitations are modeled by the generalized oscillator strengths of the constituent atoms. Using this procedure we have calculated the stopping power and the energy-loss straggling of DNA for hydrogen- and helium-ion beams at incident energies ranging from $10 \mathrm{keV} /$ nucleon to $10 \mathrm{MeV} /$ nucleon. The mean excitation energy of dry DNA is found to be $I=81.5 \mathrm{eV}$. Our present results are compared with available calculations for liquid water showing noticeable differences between these important biological materials. We have also evaluated the electron excitation probability of DNA as a function of the transferred energy by the swift projectile as well as the average energy of the target electronic excitations as a function of the projectile energy. Our results show that projectiles with energy $\lesssim 100 \mathrm{keV} /$ nucleon (i.e., around the stopping-power maximum) are more suitable for producing lowenergy secondary electrons in DNA, which could be very effective for the biological damage of malignant cells. $\odot 2011$ by Radiation Research Society

\section{INTRODUCTION}

The study of the interaction of ion beams with biological materials is very important for predicting the effects of radiation in living tissues. The relevance of this topic can be seen in its numerous applications in

\footnotetext{
1 These authors contributed equally to this work.

2 Address for correspondence: Departamento de Física - Centro de Investigación en Optica y Nanofísica ( $\mathrm{CIOyN})$, Universidad de Murcia, E-30100 Murcia, Spain; e-mail: rgm@um.es.
}

radiation microdosimetry, medical physics, therapy by heavy ions, and space radiation health $(1-3)$.

Using energetic ion beams for radiation therapy has become a promising technique because high doses can be deposited locally at tumor sites, reducing the damage to the surrounding critical organs. Hadrontherapy exploits the enhanced energy deposition taking place at the end of the range of energetic ion beams (the so-called Bragg peak) and the rapid dose fall-off beyond that depth, which can be used for maximum dose deposition within a selected tumor volume. This characteristic is not shared by other ionizing radiations such as electrons and photons, and it allows a minimization of the damage to healthy cells (4-6).

In cancer radiotherapy the selection of ion beam energies and the determination of ion ranges are crucial for the calculation of the delivered dose. There has been substantial progress in several algorithms used to calculate the depth-dose distributions for ion beams. In particular, Monte Carlo approaches enable inclusion of the relevant processes in the interaction of ion beams with inhomogeneous tissues in great detail and allow the calculation of accurate local dose distributions. Several Monte Carlo codes are available for simulating proton transport in water, which is used as a substitute for soft biological tissue, including PARTRAC (7), PTRAN (8), PETRA (9) and GEANT4 (10), among others (11). Nonetheless, the accuracy of the calculations provided by these programs is critically affected by the input values used for calculating the energy loss of the incident ion beam in the target material (12).

Most calculations of the energy loss of ions in solids are based on the Bethe theory (13), where the most important factor is the mean excitation energy of the target. At high projectile energies (in the Bethe region), the stopping powers predicted by the Bethe theory are within a few percent of the experimental data (14). However, this theory is inadequate around the Bragg peak (which corresponds to the maximum stoppingpower region) and below (15). In this case, theories more elaborate than Bethe's high-energy approximation must 
be considered for realistic calculations of the energy loss of ion beams in matter. It should be noted that medical radiation dosimetry requires accuracies of $1 \%$ on stopping powers (16).

Experimental data for the stopping of ions in matter have been compiled by Ziegler (14) and by Paul (17), and a summary of available tables and codes has been published in an ICRU Report (18).

An effort to study the interaction of energetic ion beams with liquid water at intermediate energies has been carried out recently, since water represents over $80 \%$ of the content of the cells of soft tissues (19-26). However, little work has been done concerning the electron energy loss of energetic ions in DNA targets.

Research on the effects of radiation on DNA, the most important biological material, is very active, because determining the relationship between the energy deposited by fast particles in the target and the damage they cause is important to radiation biophysics (27-30). DNA damage can be produced by direct ionization and excitation of DNA electrons, by secondary electrons generated in other parts of the cells (31), or by indirect chemical reactions of water radiolysis products with DNA (32). Even electrons with subionizing energies can cause lethal lesions in DNA $(33,34)$. Therefore, a detailed study of the energy loss of ions in biological targets (such as DNA or liquid water) is desirable to improve our understanding and modeling capabilities of the action of radiation in ion-beam cancer therapy (29, 30,35 ).

In this paper we examine the energy loss of fast proton and helium-ion beams due to electron excitations (including both continuum and discrete transitions) in dry DNA for a wide range of projectile energies (from $10 \mathrm{keV} /$ nucleon to $10 \mathrm{MeV} /$ nucleon). We apply the wellknown dielectric formalism (36) for the energy loss of swift projectiles in matter. The target electron excitation spectrum is accounted for by using a combination of Mermin-type energy-loss functions to describe the outershell electron excitations and the atomic generalized oscillator strengths for the inner-shell electron excitations. The reliability of this so-called MELF-GOS method $(37,38)$ has been proven in the description of atomic collisions with solids, for elemental or compounds targets and for light ion beams with energies covering a wide range (38-40).

\section{THEORETICAL FRAMEWORK}

A swift charged particle that moves through a medium loses energy primarily via electronic excitation of target atoms. Except at very low velocities, a heavy charged projectile loses a negligible amount (compared to electronic excitations) of energy in elastic collisions with target nuclei. Therefore, in the energy range considered here (from $10 \mathrm{keV} /$ nucleon to $10 \mathrm{MeV} /$ nucleon), we will neglect elastic energy loss in the calculations.

The electron energy loss is mainly characterized by two factors: the stopping power, $S$, and the energy-loss straggling, $\Omega^{2}$, which represent the mean and the variance of the energy lost by the projectile per unit path length, respectively.

At low and intermediate projectile energies, corresponding to the region around the maximum stopping power, it is necessary to consider the processes of electron capture and loss by the projectile, which gives a continuous charge exchange of the projectile in their path through the solid affecting its energy loss. Therefore, for a projectile with atomic number $Z_{1}$, atomic mass $M_{1}$ and energy $E$ that bombards a solid, the target stopping power and energy-loss straggling can be obtained from a weighted sum of the partial stopping powers, $S_{q}$, and energy-loss stragglings, $\Omega_{q}^{2}$, for each charge state $q$ of the projectile in the following manner (38):

$$
S(E)=\sum_{q=0}^{Z_{1}} \phi_{q}(E) S_{q}(E), \quad \Omega^{2}(E)=\sum_{q=0}^{Z_{1}} \phi_{q}(E) \Omega_{q}^{2}(E),
$$

where $\phi_{q}$ is the probability of finding the projectile in a given charge state $q$. Since charge equilibrium is reached a few femtoseconds after the projectile penetrates into the solid, we assume that $\phi_{q}$ represents the charge-state fractions at equilibrium, which depend on the target nature, the projectile and its energy. The values of $\phi_{q}$ are obtained from the parameterization made in the CasP code (41). For compound targets such as DNA, this code applies Bragg's additivity rule (42) to the target elemental constituents to find the projectile charge fractions.

We have not included in Eq. (1) the contribution of electron capture and loss to the energy loss of the projectile since cross sections for these processes are lacking for a complex target like DNA and also because estimates for simpler targets (39) are negligible at high energies and amount to $<5 \%$ for projectile energies less than $\sim 100 \mathrm{keV}$.

For a projectile with charge state $q$ moving through a solid, the energy-dependent stopping power, $S_{q}(E)$, and energy-loss straggling, $\Omega_{q}^{2}(E)$, can be written as $(36,43)$

$$
\begin{gathered}
S_{q}(E)=\int_{0}^{\infty} d(\hbar \omega) \hbar \omega P_{q}(E, \omega), \\
\Omega_{q}^{2}(E)=\int_{0}^{\infty} \mathrm{d}(\hbar \omega)(\hbar \omega)^{2} P_{q}(E, \omega),
\end{gathered}
$$

where $P_{q}(E, \omega)$ is the probability per unit path length that the particle with charge $q$ and energy $E$ transfers to the target an energy $\hbar \omega$ in an inelastic process (i.e., individual and collective electron excitations as well as ionizations) for any possible momentum transfer $\hbar k$. 
The dielectric formalism, which is based on the firstorder perturbation theory (plane-wave Born approximation) (36), provides the following expression for $P_{q}(E, \omega)$ :

$$
P_{q}(E, \omega)=\frac{M_{1} e^{2}}{\pi \hbar^{2} E} \int_{k_{\min }}^{\infty} \frac{\mathrm{d} k}{k} \rho_{q}^{2}(k) \operatorname{Im}\left[\frac{-1}{\varepsilon(k, \omega)}\right]
$$

where $k_{\min }=\omega / \sqrt{\left(2 E / M_{1}\right)}, e$ is the absolute value of the electron charge, $\rho_{q}(k)$ is the Fourier transform of the projectile charge density for the charge state $q$, and $\varepsilon(k, \omega)$ is the dielectric function of the target.

\section{Energy-Loss Function of Dry DNA}

The key parameter to obtain reliable results for the energy losses above is the energy-loss function (ELF) of the material, $\operatorname{Im}[-1 / \varepsilon(k, \omega)]$, since it contains all the information about the electron excitation spectrum of the target. Thus it is essential to use a good description of the target ELF for the whole $k-\omega$ plane (that is, the Bethe surface).

The well-known Lindhard dielectric function is applicable to only a limited number of so-called nearly-free-electron materials, like aluminum. For other targets, a commonly used method for obtaining the ELF is to employ the experimental energy-loss function at $k=0$ (optical limit) and extend it to $k>0$ by introducing suitable dispersion relationship schemes [see ref. (44) for a discussion].

For dry DNA films, Inagaki et al. (45) published experimental optical data based on reflectance measurements for photon energies ranging from 2 to $82 \mathrm{eV}$. As in ref. (45), we assume that the mass density of DNA is $1.35 \mathrm{~g} / \mathrm{cm}^{3}$ and that the typical nucleotide is composed of a thymine-adenine pair plus two phosphate groups and two sugars (46), so the target molecular formula is $\mathrm{C}_{20} \mathrm{H}_{27} \mathrm{~N}_{7} \mathrm{O}_{13} \mathrm{P}_{2}$.

We model the ELF of dry DNA films using the MELF-GOS method $(37,38)$. This approach allows the separation of contributions from the outer electron and the inner-shell electron excitations to the target ELF, namely,

$$
\operatorname{Im}\left[\frac{-1}{\varepsilon(k, \omega)}\right]=\operatorname{Im}\left[\frac{-1}{\varepsilon(k, \omega)}\right]_{\text {outer }}+\operatorname{Im}\left[\frac{-1}{\varepsilon(k, \omega}\right]_{\text {inter }} .
$$

The excitations of the outer electrons of the solid, including both collective and single-particle excitations, are described by fitting the experimental optical ELF $(k$ $=0)$ to a linear combination of Mermin-type ELFs,

$$
\begin{aligned}
& \operatorname{Im}\left[\frac{-1}{\varepsilon(k, \omega)}\right]_{\text {outer }}=\operatorname{Im}\left[\frac{-1}{\varepsilon(k=0, \omega)}\right]_{\exp } \\
& \quad=\sum_{i} A_{i} \operatorname{Im}\left[\frac{-1}{\varepsilon_{\mathrm{M}}\left(\omega_{i}, \gamma_{i}, k=0, \omega\right)}\right] \Theta\left(\omega-\omega_{\mathrm{th}, i}\right),
\end{aligned}
$$

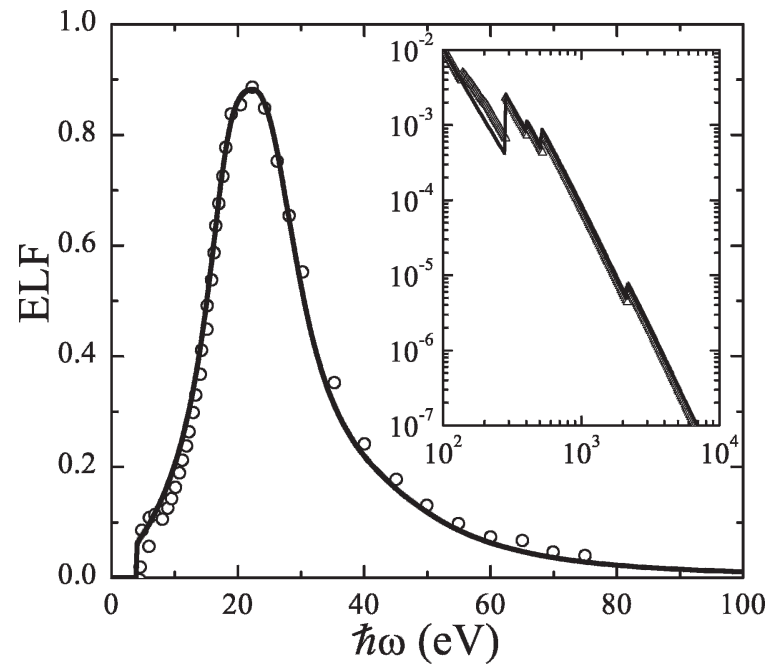

FIG. 1. ELF of dry DNA in the optical limit $(k=0)$ as a function of the transferred energy, $\hbar \omega$. Solid curves correspond to the MELFGOS model, while circles represent experimental data from Inagaki et al. (45). The inset shows the ELF at high transferred energies in a log$\log$ plot, with triangles representing the results derived from X-ray atomic scattering factors (48).

where $\varepsilon_{\mathrm{M}}$ is the Mermin dielectric function (47). The fitting parameters $\omega_{i}$ and $\gamma_{i}$ are related to the position and width, respectively, of the $i$ th Mermin-type ELF, while the coefficients $A_{i}$ are the corresponding weights. The threshold energy $\hbar \omega_{\mathrm{th}, i}$ for DNA films has a value of $4 \mathrm{eV}(45)$.

On the other hand, inner-shell electrons retain their atomic character since they have large binding energies; therefore, they are suitably modeled in terms of the generalized oscillator strengths (GOS). The connection between the ELF and the GOS model is given by

$$
\operatorname{Im}\left[\frac{-1}{\varepsilon(k, \omega)}\right]_{\text {inner }}=\frac{2 \pi^{2} N}{\omega} \sum_{n l} \frac{\mathrm{d} f_{n l}(k, \omega)}{\mathrm{d} \omega},
$$

where $\mathrm{d} f_{n 1}(k, \omega)$ is the GOS of the $(n, l)$ sub-shell and $N$ is the molecular density of the target (for dry DNA, $N=1.28 \cdot 10^{-3}$ molecule $\left./ \AA^{3}\right)$. We use the hydrogenic approach to obtain the GOS because it is analytical and describes the contribution of the K-shell ionization corresponding to C, N, O and P atoms well (38). Thus 84 core electrons of each DNA nucleotide are modeled by the GOS, Eq. (6), while 246 electrons are treated as outer electrons, Eq. (5).

In Fig. 1 we show the optical ELF of solid DNA as a function of the transferred energy, $\hbar \omega$. The circles are experimental results from Inagaki et al. (45) obtained in the extreme ultraviolet region; the triangles in the inset correspond to the experimental data obtained from Xray atomic scattering factors (48), and they describe the ELF at large transferred energies. The ELF also shows peaks at about 284, 410, 543 and $2146 \mathrm{eV}$ that correspond to the $\mathrm{K}$-shell edges for carbon, nitrogen, oxygen and phosphorus, respectively (49). The solid 
TABLE 1

Parameters Used to Fit the Outer Electron Excitations Contribution to the ELF (see Eq. 5) of Dry DNA Films whose Molecular Density is $N=1.28 \cdot 10^{-3}$ molecule $/ \AA^{3}$

\begin{tabular}{cccl}
\hline$i$ & $\hbar \omega_{i}(\mathrm{eV})$ & $\hbar \gamma_{i}(\mathrm{eV})$ & \multicolumn{1}{c}{$A_{i}$} \\
\hline 1 & 19.0 & 9.0 & 0.159 \\
2 & 25.2 & 15.0 & 0.397 \\
3 & 43.0 & 35.0 & 0.0707 \\
\hline
\end{tabular}

Note. We used $\hbar \omega_{\mathrm{th}, i}=4 \mathrm{eV}$ for all values of $i$.

curves correspond to our fit according to the MELFGOS method, including the contribution of both the outer and the inner electrons. The ELF of dry DNA is described by a sum of three Mermin ELF for the outer electrons (with the parameters given in Table 1) and by a GOS for each one of the C, N, O and P K-shells, as shown in Fig. 1; each inner shell is visible as a sharp rise in the ELF at the corresponding threshold energy.

One of the advantages of the MELF-GOS method is that the fit of the ELF in the optical limit is analytically and automatically extended to $k \neq 0$ through the properties of the Mermin dielectric function and the GOS model $(38,50)$; therefore, it is not necessary to assume a particular ELF dependence on the momentum transfer. For the materials where experimental data are available for the ELF at $k \neq 0$, the MELF-GOS reproduces the experimental ELF well $(25,44,50,51)$. Therefore, we use the procedure described previously to extend the optical ELF to the whole Bethe surface.

The resulting ELF should satisfy the $f$-sum rule for any wave number $k$. Thus the effective number of electrons per molecule $N_{\text {eff }}$ that may be excited up to a maximum transferred energy $\hbar \omega$ from an incident projectile,

$$
N_{\mathrm{eff}}(\omega)=\frac{m_{\mathrm{e}}}{2 \pi^{2} e^{2} N} \int_{0}^{\omega} d \omega^{\prime} \omega^{\prime} \operatorname{Im}\left[\frac{-1}{\varepsilon\left(k, \omega^{\prime}\right)}\right],
$$

must tend toward the target atomic number as the transferred energy $\hbar \omega$ goes to infinity. Here $m_{\mathrm{e}}$ represents the electron mass. The solid line in Fig. 2 shows the effective number of excited electrons $N_{\text {eff }}$ as a function of the transferred energy $\hbar \omega$ at zero momentum for dry DNA. The contribution due to the outer electrons of DNA represents $77.2 \%$ of the total effective number of electrons; the remaining contribution comes from the $\mathrm{K}$ shells of C (11.37\%), N (3.7\%), O (6.83\%) and P (0.91\%). The total number of electrons in a DNA nucleotid is 330 , which is indicated by an arrow in Fig. 2.

We also check that the ELF of dry DNA obtained using the MELF-GOS model satisfies the KramersKronig (KK) (or perfect screening) sum rule (52),

$$
\mathrm{KK}(\omega)=\frac{2}{\pi} \int_{0}^{\omega} d \omega^{\prime} \frac{1}{\omega^{\prime}} \operatorname{Im}\left[\frac{-1}{\varepsilon\left(k=0, \omega^{\prime}\right)}\right]+n^{-2}(0),
$$

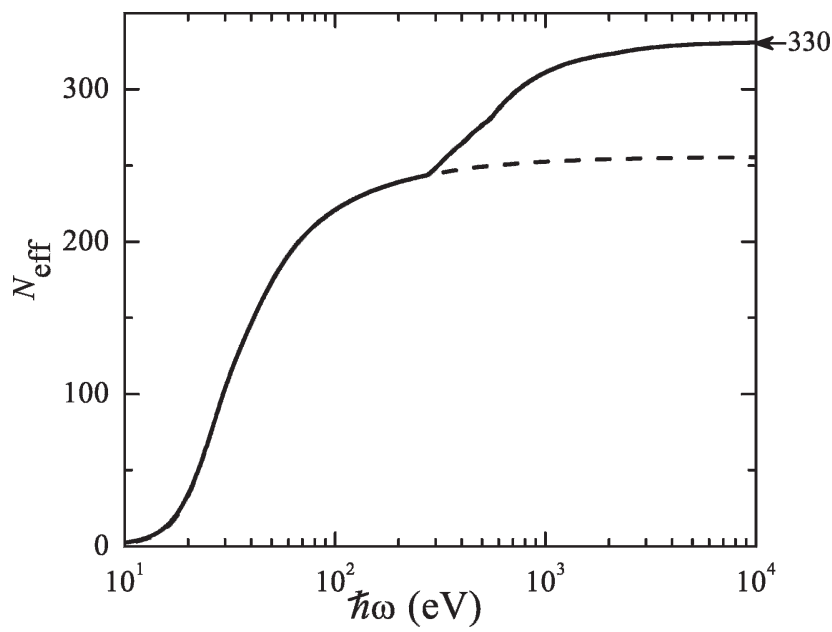

FIG. 2. Effective number of electrons of dry DNA as a function of the maximum transferred energy, $\hbar \omega$. Solid line represents the contribution of all the electrons, while the dashed line is the contribution of the outer-shell electrons. The arrow indicates the total number of electrons in a DNA nucleotide.

which must go to unity when $\hbar \omega \rightarrow \infty$. For the refractive index at low energies, $n(0)$, we used the value 1.58 (45). This sum rule is an important test for the accuracy of our fit at low transferred energies, and it is fulfilled to better than $99 \%$.

\section{RESULTS AND DISCUSSION}

Accurate values of the mean excitation energy $I$ for biological materials such as DNA are desirable because a difference in the $I$ value of only a few percent might cause sizable changes in the range and stopping maximum of therapeutic ion beams $(12,53)$, which could be biologically relevant for the accuracy of the energy deposited in nanometer volumes (54).

The MELF-GOS method allows an accurate description of the dielectric properties of dry DNA including information regarding the condensed state, such as electronic screening and collective effects. In this context we can evaluate the cumulative mean excitation energy $I(\omega)$ of the target as a function of the transferred energy $\hbar \omega$ as follows:

$$
\ln [I(\omega)]=\frac{\int_{0}^{\omega} d \omega^{\prime} \omega^{\prime} \ln \left(\omega^{\prime}\right) \operatorname{Im}\left[-1 / \varepsilon\left(k=0, \omega^{\prime}\right)\right]}{\int_{0}^{\omega} d \omega^{\prime} \omega^{\prime} \operatorname{Im}\left[-1 / \varepsilon\left(k=0, \omega^{\prime}\right)\right]} .
$$

The cumulative mean excitation energy allows us to calculate how electrons in different shells contribute to the mean excitation energy $I$, which is obtained from Eq. (9) when the transfer energy $\hbar \omega \rightarrow \infty$. In Fig. 3 we show the value of $I(\omega)$ of dry DNA as a function of the transferred energy. When $\hbar \omega \rightarrow \infty$ we obtain $I=81.5 \mathrm{eV}$, where $43.5 \mathrm{eV}$ is the outer electron contribution. The 


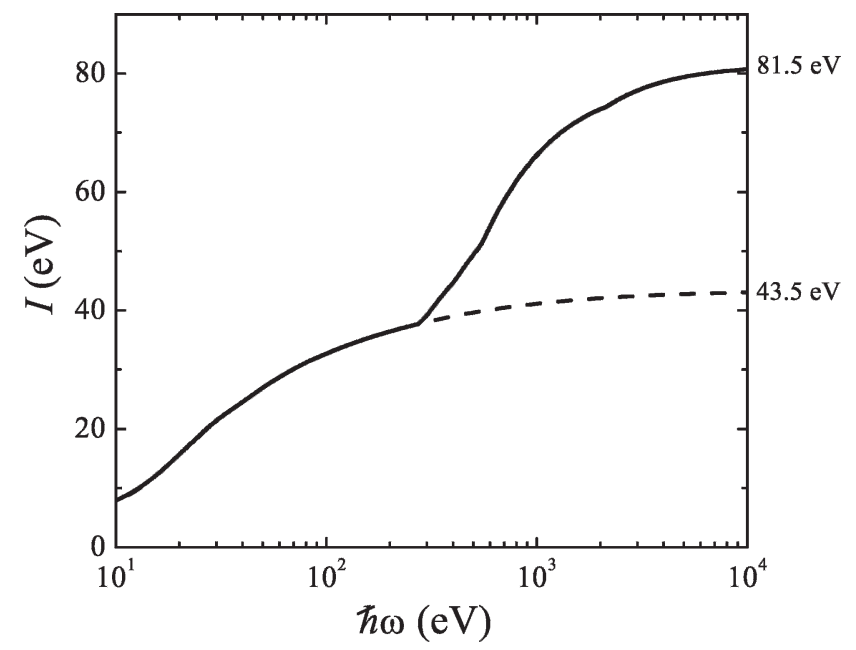

FIG. 3. Cumulative mean excitation energy of a dry DNA target as a function of the energy transferred, $\hbar \omega$. Solid line represents the contribution of all the electrons, while the dashed line is due only to the outer-shell electrons.

graph clearly shows how the atomic shell structure is evident in the cumulative mean excitation energy and illustrates that outer- and inner-shell electrons contribute to the mean excitation energy in almost the same proportion. The value of $I$ we obtained is somewhat higher than that of $77.9 \mathrm{eV}$ calculated previously by LaVerne and Pimblott (46) and clearly differs from that of $\sim 85 \mathrm{eV}$ obtained when applying Bragg's rule following the procedure outlined in ICRU Report 49 (55) as well as from that of $86.64 \mathrm{eV}$ calculated by Tan et al. (23). Although the same experimental ELF was used by different authors, the discrepancies in the calculated $I$ values could be due to the extrapolation of the ELF at intermediate energy transfers used by each research group, where there are no experimental data. It is also interesting to compare with the mean excitation energy of liquid water, where a value of $I_{\text {liq.water }}=79.4 \mathrm{eV}$ was recently obtained (25) using the MELF-GOS method.

From the theoretical model presented in the previous section, based on the dielectric formalism and on a realistic description of the electron properties of dry DNA, we have calculated the probability to produce electronic excitations of energy $\hbar \omega$ per unit path length, $P_{q}(E, \omega)$, by a given projectile with charge $q$ and incident energy $E$. In our calculations the electron density $\rho_{q}$ for each charge state of the projectile was described using the Brandt-Kitagawa statistical model (56). In Fig. 4 we show the results of $P_{q}(E, \omega)$ corresponding to $\mathrm{H}^{+}(q=1)$ at $E=25 \mathrm{keV}, 100 \mathrm{keV}, 500 \mathrm{keV}, 1 \mathrm{MeV}$ and $5 \mathrm{MeV}$ and for neutral projectiles $\mathrm{H}^{0}$ at $E=25 \mathrm{keV}$ and $100 \mathrm{keV}$; these energies were chosen as representatives for the more probable charge states. We observe that the most probable electronic excitations have energies in the range $\sim 10-30 \mathrm{eV}$, particularly for projectile energies below and around $100 \mathrm{keV}$. The maximum value of $P_{q}(E, \omega)$, that is, the maximum probability to excite

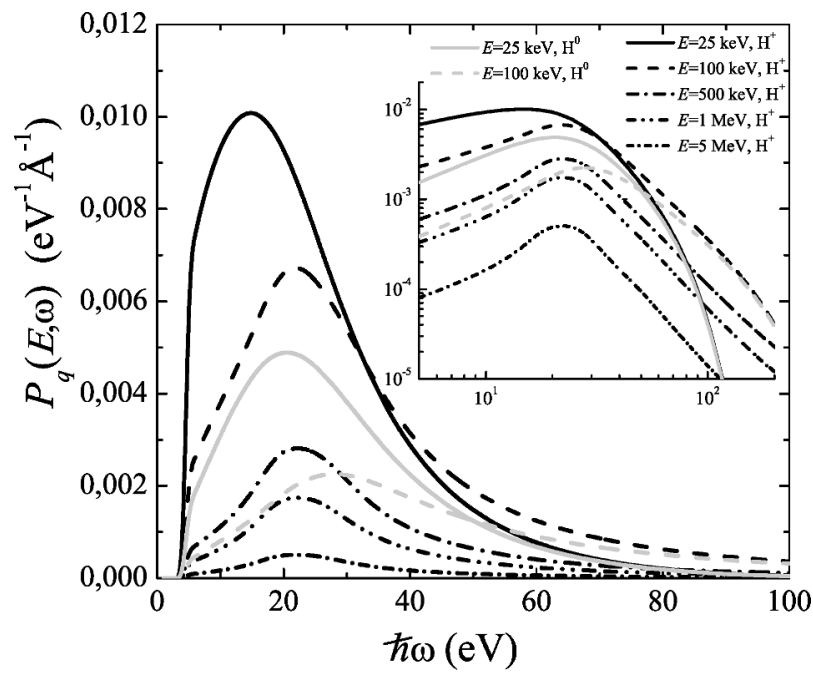

FIG. 4. Probability $P_{q}(E, \omega)$ per unit path length that a particle, with charge $q$ and energy $E$, loses an energy $\hbar \omega$ into electronic excitations of dry DNA. We have represented the results for a proton $(q=1)$ at energies $E=25 \mathrm{keV}$ (black solid line), $100 \mathrm{keV}$ (black dashed line), $500 \mathrm{keV}$ (black dash-dotted line), $1 \mathrm{MeV}$ (black dashdot-dotted line) and $5 \mathrm{MeV}$ (black short dash-dotted line) for a hydrogen particle $(q=0)$ at $E=25 \mathrm{keV}$ (gray solid line) and $100 \mathrm{keV}$ (gray dashed line). To appreciate the behavior of the tails, the inset shows the same results in a log-log scale for a larger region of $\hbar \omega$.

electrons decreases as the projectile energy $E$ increases. The probability $P_{q}(E, \omega)$ is smaller for neutral than for charged isoenergetic projectiles, although at $\hbar \omega \gtrsim 100 \mathrm{eV}$ both excitation probabilities practically coincide. These results are important because although the damage in DNA can be produced by direct ionization of inner shells ( $\hbar \omega>284 \mathrm{eV}$ for the $\mathrm{K}$-shell of $\mathrm{C}$ ), recent reports point to electrons with sub-ionizing energies as the ones responsible for producing lethal damage in DNA $(30,33,34)$.

In Fig. 4 we also observe that the probability to produce electronic excitations due to $\mathrm{H}^{0}$ projectiles is lower than for protons, even when its maximum value is shifted toward higher electron energies, compared to the same proton energies. However, the fraction of $\mathrm{H}^{0}$ in the incident beam is small, as given by the CasP code (41), representing $\sim 65, \sim 10$ and $\sim 2 \%$ at 25,100 and $150 \mathrm{keV}$, respectively.

The average energy transferred to DNA by electronic excitations by a projectile with energy $E, W_{\text {average }}(E)$, can be calculated as

$$
W_{\text {average }}(E)=\frac{\sum_{q=0}^{Z_{1}} \phi_{q}(E) \int d(\hbar \omega)(\hbar \omega) P_{q}(E, \omega)}{\sum_{q=0}^{Z_{1}} \phi_{q}(E) \int d(\hbar \omega) P_{q}(E, \omega)} .
$$

Figure 5 shows $W_{\text {average }}(E)$ as a function of the energy of hydrogen and helium projectiles. We can see that $W_{\text {average }}$ $(E)$ is very similar in the two cases. The energy loss of hydrogen- and helium-ion beams will not follow the 


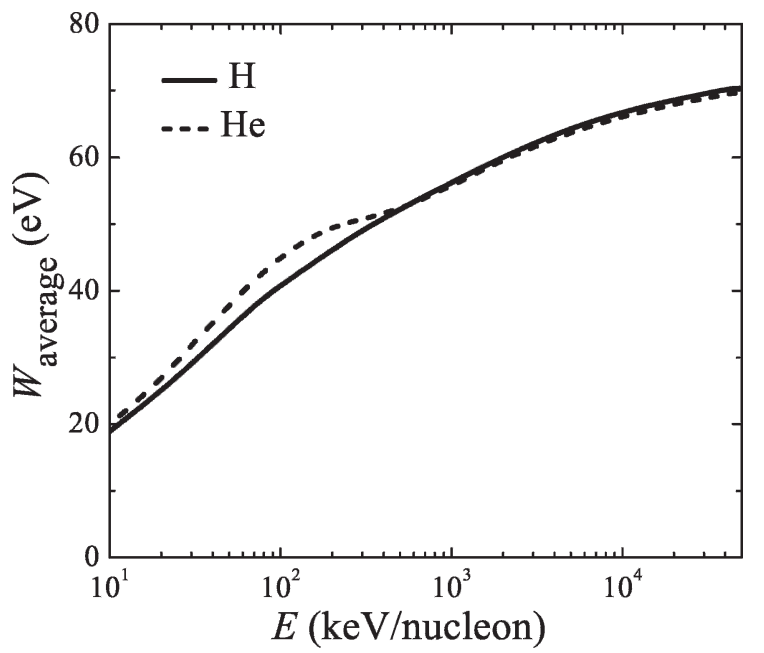

FIG. 5. Average energy transferred to electronic excitations induced in DNA by incident hydrogen and helium particles as a function of their energy $E$.

same trend as $W_{\text {average }}(E)$ because the number of electron excitations induced in the medium, determined by $P_{q}(E, \omega)$, decreases with projectile energy. When $E=$ $10 \mathrm{keV} /$ nucleon the average energy of the excitations is around $20 \mathrm{eV}$, increasing to $70 \mathrm{eV}$ for $E=50 \mathrm{MeV} /$ nucleon. We can estimate the energy of the secondary electrons resulting from the electronic excitations as $W_{\text {average }}$ $-E_{\text {bind }}$, where $E_{\text {bind }} \sim 10 \mathrm{eV}$ is the binding energy (57-59); these electrons are energetic enough to produce strand breaks in DNA due to the low threshold energy for radiation damage in biomolecules (60). The average energy of the secondary electrons produced in DNA is similar to the same magnitude when evaluated in water (29).

The calculated stopping power of dry DNA for proton and $\alpha$-particle beams is shown as a function of the projectile energy in Figs. 6 and 7, respectively. The maximum values of $S$ are around $100 \mathrm{keV}$ for protons and $120 \mathrm{keV} /$ nucleon for $\alpha$-particle beams; these energies also correspond to the maximum probability to excite target electrons, as discussed previously in relation to Fig. 4. Symbols in Fig. 6 represent the calculations of Tan et al. (23), which were also evaluated applying the dielectric formalism to the optical ELF of DNA (45) but with a different scheme to extend them to $k \neq 0$ values. Because liquid water represents the most relevant material in biological materials, for comparison purposes we also show the stopping power of liquid water obtained by a procedure similar to the one described elsewhere (25). Both targets present the maximum of the stopping power at the same projectile energy, although the stopping-power values for liquid water are always lower than for dry DNA (15\% at $10 \mathrm{keV}$ and $\sim 20 \%$ at high energy). This different behavior of DNA and liquid water regarding the energy loss of fast particles is due mainly to their different excitation spectra and, to a lesser extent, to their different electron density $\left(N_{\mathrm{DNA}}^{e}=0.412 \mathrm{e} / \AA^{3}, N_{\text {liq.water }}^{e}=0.334 \mathrm{e} / \AA^{3}\right)$.

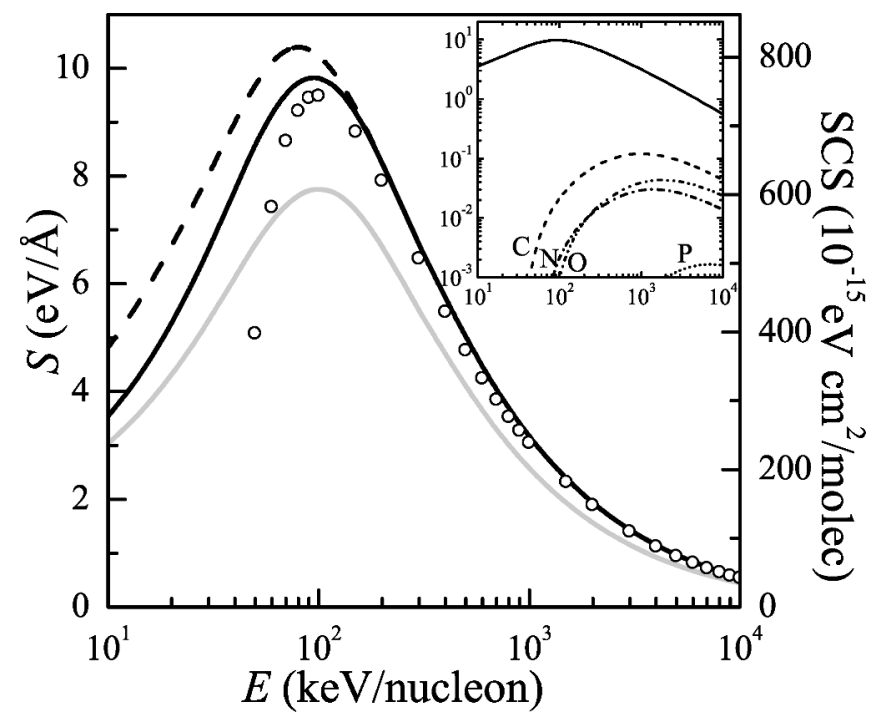

FIG. 6. Stopping power of dry DNA (black solid line) for a hydrogen-ion beam as a function of the incident energy; the right axis corresponds to the stopping cross section. The stopping power of DNA for a frozen-charge proton is shown as a dashed line. For comparison purposes, the stopping power of liquid water (gray solid line) (25) is also shown. Symbols represent data from ref. (23). The inset shows the $\mathrm{K}$-shell contributions to the total stopping power (solid line) in a log-log plot: $\mathrm{C}$ (dashed line), $\mathrm{N}$ (dash-dot line), $\mathrm{O}$ (dash-dot-dot line) and P (short-dash-dot line) of dry DNA.

At low projectile energies (around and below the stopping-power maximum) the process of electron capture and loss by the projectile becomes important. The equilibrium charge-state fractions, $\phi_{q}$, of the projectile that enter in our calculations through Eq. (1) are obtained from the CasP code (41) by interpolation of the values corresponding to DNA's atomic constituents. To show the influence of the charge state in our calculations, in Figs. 6 and 7 we also show the stopping power of DNA without considering the change in the charge state (i.e. frozen-charge approximation) of protons and $\alpha$ particles.

The insets of Figs. 6 and 7 show the contributions of the $\mathrm{K}$-shells of $\mathrm{C}, \mathrm{N}, \mathrm{O}$ and $\mathrm{P}$ to the stopping power of dry DNA for hydrogen- and helium-ion beams, respectively. As can be seen, the dominant contribution to the stopping power comes from the outer target electrons, but when the incident projectile energy increases, the contribution from inner shells becomes more important, representing $15 \%$ of the total energy loss at projectile energies of $10 \mathrm{MeV} /$ nucleon.

The energy loss of a charged particle in a material is a stochastic phenomenon; therefore, it is important to characterize both the stopping power and the energyloss straggling (Eq. 2). In Fig. 8 we show the energy-loss straggling $\Omega^{2}$ of dry DNA for hydrogen and helium projectiles as a function of their energy. In the inset of Fig. 8 we show the contributions of the K-shells of C, N, $\mathrm{O}$ and $\mathrm{P}$ to the energy-loss straggling, which is larger than those to the stopping power, representing $31 \%$ of 


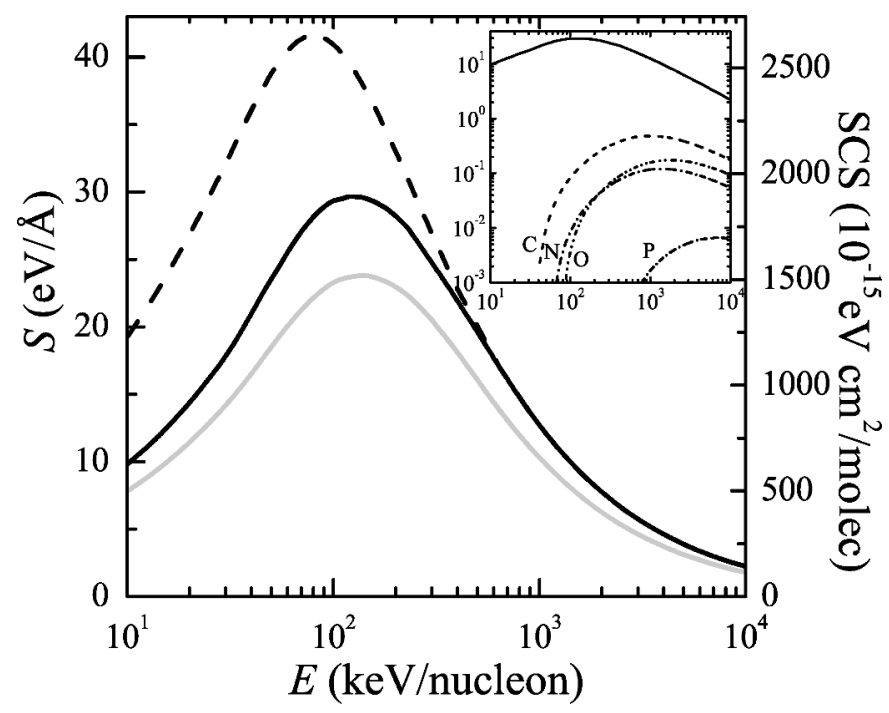

FIG. 7. Stopping power of dry DNA (black solid line) for an $\alpha$ particle beam as a function of the incident energy; the right axis corresponds to the stopping cross section. The stopping power of DNA for a frozen-charge proton is shown as a dashed line. For comparison purposes, the stopping power of liquid water (gray solid line) (25) is also shown. The inset shows the K-shell contributions to the total stopping power (solid line) in a log-log plot: $\mathrm{C}$ (dashed line), $\mathrm{N}$ (dash-dot line), O (dash-dot-dot line) and $\mathrm{P}$ (short-dash-dot line) of dry DNA.

the total straggling at a projectile energy of $10 \mathrm{MeV} /$ nucleon. Therefore, we conclude that a proper description of inner-shell contributions to the ELF using the GOS of the target elements is important for energy-loss calculations of high-energy projectiles, mostly for the energy-loss straggling.

\section{CONCLUSIONS}

We have applied the dielectric formalism to calculate the main significant magnitudes in the energy loss of hydrogen- and helium-ion ion beams in a dry DNA target, namely, the probability of electronic excitations, the stopping power, and the energy-loss straggling. The calculations have been done taking into account the charge-state fractions of the projectile, which vary as a function of the incident energy. The excitation spectrum of the DNA target has been described accurately by means of the MELF-GOS method $(37,38)$, which uses Mermin-type energy-loss functions for the outer electron excitations and generalized oscillator strengths for the (preserving atomic character) inner-shell excitations.

We found a mean excitation energy $I=81.5 \mathrm{eV}$, which is between the values reported previously $(23,46)$. By analyzing the energy distribution of the electron excitations produced by $\mathrm{H}^{+}$and $\mathrm{H}^{0}$ in dry DNA for several projectile energies, we conclude that protons with energies $\lesssim 100 \mathrm{keV}$ are very efficient in producing low-energy secondary electrons, which despite their low energy are still able to produce strand breaks and

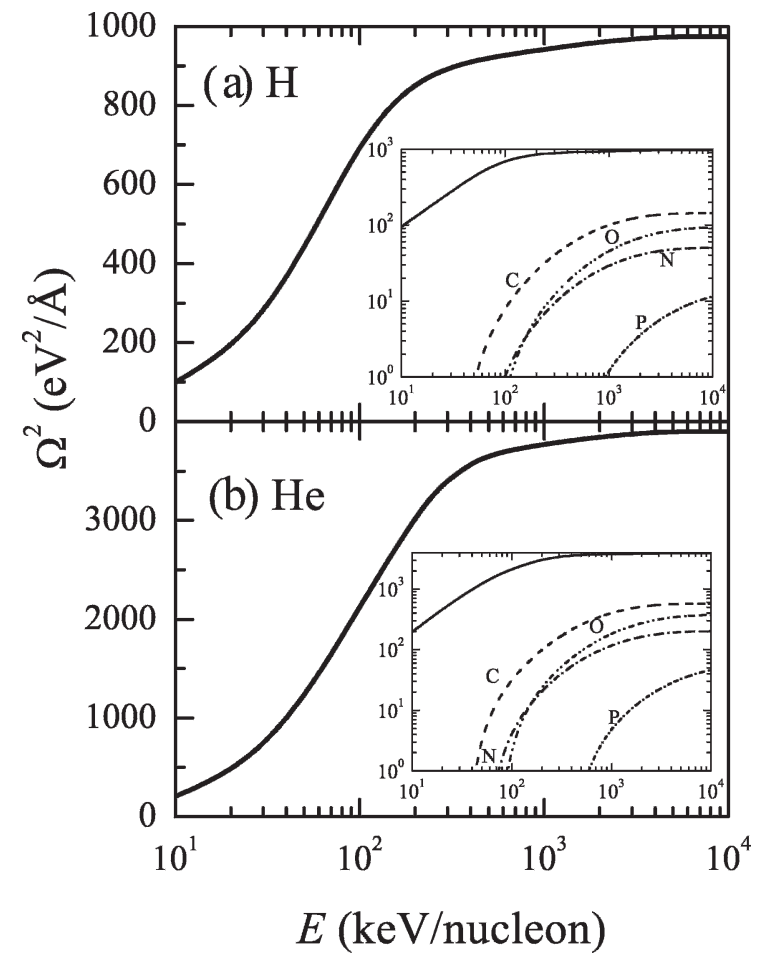

FIG. 8. Energy-loss straggling of dry DNA for (panel a) hydrogen- and (panel b) helium-ion beams as a function of the incident energy. The discontinuous lines in the inset represent the contribution coming from the $\mathrm{K}$-shell of $\mathrm{C}$ (dashed line), $\mathrm{N}$ (dash-dot line), $\mathrm{O}$ (dash-dot-dot line) and $\mathrm{P}$ (short-dash-dot line).

therefore DNA damage according to both experimental evidence $(33,34)$ and theoretical considerations $(30)$. These proton energies are lower or around the one that produces the maximum value of the stopping power.

We have calculated the stopping power and the energy-loss straggling of dry DNA for hydrogen and helium-ion beams for a broad range of incident energies (from $10 \mathrm{keV} /$ nucleon to $10 \mathrm{MeV} /$ nucleon). Since there are no experimental data for the energy loss of light ions in dry DNA, we compared our results with analogous calculations for liquid water (25). The stopping-power maximum of DNA is found at the nearly the same projectile energy as that for liquid water. On the other hand, the stopping power of DNA is $\sim 20 \%$ larger than that for liquid water. The present results are expected to be useful to Monte Carlo simulation of proton and $\alpha$ particle transport and energy loss in biological tissues.

\section{ACKNOWLEDGMENTS}

This work has been supported financially by the Spanish Ministerio de Ciencia e Innovación (projects FIS2006-13309-C02-01 and FIS2006-13309-C02-02). CDD thanks the Spanish Ministerio de Ciencia e Innovación and Generalitat Valenciana for support under the Ramón y Cajal Program. IK and DE acknowledge financial support from the Research Committee of the University of Ioannina (Grant no. 80037) and the European Union FP7 ANTICARB (HEALTH-F2-2008-201587). 
Received: January 15, 2010; accepted: July 7, 2010; published online: November 1, 2010

\section{REFERENCES}

1. H. Breuer and B. J. Smit, Proton Therapy and Radiosurgery. Springer, Berlin, 2000.

2. J. E. Turner, Atoms, Radiation, and Radiation Protection. Wiley$\mathrm{VCH}$, Weinheim, 2007.

3. M. Durante and F. A. Cucinotta, Heavy ion carcinogenesis and human space exploration. Nat. Rev. Cancer 8, 465-472 (2008).

4. G. Kraft, Tumor therapy with heavy charged particles. Prog. Particle Nucl. Phys. 45, S473-S544 (2000).

5. O. Jäkel, Hadron therapy: Radiotherapy using fast ion beams. In Ion Beam Science: Solved and Unsolved Problems, Part I (P. Sigmund, Ed.), pp. 37-57. Matematisk-fysiske Meddelelser 52, Copenhagen, 2006.

6. H. Nikjoo, S. Uehara, D. Emfietzoglou and A. Brahme, Heavy charged particles in radiation biology and biophysics. New $J$. Phys. 10, 075006-1-28 (2008).

7. W. Friedland, P. Jacob, P. Bernhardt, H. G. Paretzke and M. Dingfelder, Simulation of DNA damage after proton irradiation. Radiat. Res. 159, 401-410 (2003)

8. M. J. Berger, Proton Monte Carlo transport programme PTRAN. Report NISTIR-5113, National Institute of Standards and Technology. Gaithersburg, MD, 1993. Available online at http://www.nea.fr/tools/abstract/detail/ccc-0618.

9. J. Medin and P. Andreo, Monte Carlo calculated stopping-power ratios, water/air, for clinical proton dosimetry (50-250 MeV). Phys. Med. Biol. 42, 89-105 (1997).

10. I. Pshenichnov, I. Mishustin and W. Greiner, Distributions of positron-emitting nuclei in proton and carbon-ion therapy studied with GEANT4. Phys. Med. Biol. 51, 6099-6112 (2006).

11. H. Nikjoo, S. Uehara, D. Emfietzoglou and F. A. Cucinotta, Track-structure codes in radiation research. Radiat. Meas. 41, 1052-1074 (2006).

12. H. Paul, O. Geithner and O. Jäkel, The influence of stopping powers upon dosimetry for radiation therapy with energetic ions. Adv. Quantum Chem. 52, 292-308 (2007).

13. M. Inokuti, Inelastic collisions of fast charged particles with atoms and molecules-Bethe theory revisited. Rev. Mod. Phys. 43, 297-347 (1971).

14. J. F. Ziegler, J. P. Biersack and M. D. Ziegler, SRIM. The Stopping and Range of Ions in Matter. Available online at http:// www.srim.org.

15. P. Sigmund, Light-ion stopping near the maximum. Nucl. Instrum. Methods B 85, 541-550 (1994).

16. D. I. Thwaites, Review of stopping powers in organic materials Nucl. Instrum. Methods B 27, 293-300 (1987).

17. H. Paul, Stopping power for light ions. Available online at http:// www.exphys.uni-linz.ac.at/stopping/.

18. ICRU, Stopping Ions Heavier Than Helium. Report 73, International Commission on Radiation Units and Measurements, Bethesda, MD, 2005.

19. M. Dingfelder, M. Inokuti and H. G. Paretzke, Inelastic-collision cross sections of liquid water for interactions of energetic protons. Radiat. Phys. Chem. 59, 255-275 (2000).

20. A. Akkerman, A. Breskin, R. Chechik and Y. Lifshitz, Calculation of proton stopping power in the region of its maximum value for several organic materials and water. Radiat. Phys. Chem. 61, 333-335 (2001).

21. D. Emfietzoglou, M. Moscovitch and A. Pathak, Inelastic crosssections of energetic protons in liquid water calculated by model dielectric functions and optical data. Nucl. Instrum. Methods B 212, 101-109 (2003).
22. D. Emfietzoglou, A. Pathak, G. Papamichael, K. Kostarelos, S. Dhamodaran, N. Sathish and M. Moscovitch, A study on the electronic stopping of protons in soft biological matter. Nucl. Instrum. Methods B 242, 55-60 (2006).

23. Z. Tan, Y. Xia, M. Zhao and X. Liu, Proton stopping power in a group of bioorganic compounds over the energy range of 0.05 $10 \mathrm{MeV}$. Nucl. Instrum. Methods B 248, 1-6 (2006).

24. D. Emfietzoglou, A. Pathak and H. Nikjoo, Monte-Carlo study of energy deposition by heavy charged particles in sub-cellular volumes. Radiat. Prot. Dosimetry 126, 97-100 (2007).

25. R. Garcia-Molina, I. Abril, C. D. Denton, S. Heredia-Avalos, I. Kyriakou and D. Emfietzoglou, Calculated depth-dose distributions for $\mathrm{H}^{+}$and $\mathrm{He}^{+}$beams in liquid water. $\mathrm{Nucl}$. Instrum. Methods B 267, 2647-2652 (2009).

26. D. Emfietzoglou, R. Garcia-Molina, I. Kyriakou, I. Abril and H. Nikjoo, A dielectric response study of the electronic stopping power of liquid water for energetic protons and a new $I$-value for water. Phys. Med. Biol. 54, 3451-3472 (2009).

27. H. Nikjoo, P. O'Neill, W. E. Wilson and D. T. Goodhead, Computational approach for determining the spectrum of DNA damage induced by ionizing radiation. Radiat. Res. 156, 577-583 (2001).

28. W. Friedland, P. Jacob, P. Bernhardt, H. G. Paretzke and M. Dingfelder, Simulation of DNA damage after proton irradiation. Radiat. Res. 159, 401-410 (2003).

29. E. Surdutovich, O. I. Obolensky, E. Scifoni, I. Pshenichnov, I Mishustin, A. V. Solov'yov and W. Greiner, Ion-induced electron production in tissue-like media and DNA damage mechanisms. Eur. Phys. J. D 51, 63-71 (2009).

30. A. V. Solov'yov, E. Surdutovich, E. Scifoni, I. Mishustin and W. Greiner, Physics of ion beam cancer therapy: A multiscale approach. Phys. Rev. E 79, 011909-1-7 (2009).

31. Z. Cai and P. Coutier, Comparison between X-ray photon and secondary electron damage to DNA in vacuum. J. Phys. Chem. B 109, 4796-4800 (2005).

32. C. Chatgilialoglu and P. O'Neill, Free radicals associated with DNA damage. Exp. Gerontol. 36, 1459-1471 (2001).

33. B. Boudaïfa, P. Cloutier, D. Hunting, M. A. Huels and L. Sanche, Resonant formation of DNA strand breaks by lowenergy (3 to $20 \mathrm{eV}$ ) electrons. Science 287, 1658-1660 (2000).

34. M. A. Huels, B. Boudaïffa, P. Cloutier, D. Hunting and L. Sanche, Single, double, and multiple double strand breaks induced in DNA by $3-100 \mathrm{eV}$ electrons. J. Am. Chem. Soc. 125, 4467-4477 (2003)

35. C. Di Pietro, S. Piro, G. Tabbi, M. Ragusa, V. Di Pietro, V. Simmitti, F. Cuda, M. Anello, U. Consoli and M. Purrello, Cellular and molecular effects of protons: Apoptosis induction and potential implications for cancer therapy. Apoptosis 11, 5766 (2006).

36. J. Lindhard, On the properties of a gas of charged particles. $K$. Dan. Vidensk. Selsk. Mat. Fys. Medd. 28 (8) (1954).

37. I. Abril, R. Garcia-Molina, C. D. Denton, F. J. Pérez-Pérez and N. R. Arista, Dielectric description of wakes and stopping powers in solids. Phys. Rev. A 58, 357-366 (1998).

38. S. Heredia-Avalos, R. Garcia-Molina, J. M. Fernández-Varea and I. Abril, Calculated energy loss of swift $\mathrm{He}, \mathrm{Li}, \mathrm{B}$, and $\mathrm{N}$ ions in $\mathrm{SiO}_{2}, \mathrm{Al}_{2} \mathrm{O}_{3}$, and $\mathrm{ZrO}_{2}$. Phys. Rev. A 72, 052902-1-9 (2005).

39. S. Heredia-Avalos, J. C. Moreno-Marìn, I. Abril and R. GarciaMolina, Energy loss of $\mathrm{H}^{+}$and $\mathrm{He}^{+}$in the semiconductors $\mathrm{GaAs}$, ZnSe, InP and SiC. Nucl. Instrum. Methods B 230, 118-124 (2005).

40. R. Garcia-Molina, I. Abril, C. D. Denton and S. Heredia-Avalos, Electronic energy loss of swift $\mathrm{H}^{+}$and $\mathrm{He}^{+}$ions in solids with material science applications. Nucl. Instrum. Methods B 249, 29 33 (2006).

41. P. L. Grande and G. Schiwietz, CasP. Convolution approximation for swift particles, version 3.2, 2007. Code available at http://www. 
helmholtz-berlin.de/media/media/spezial/people/schiwietz/html/ casp.html.

42. W. H. Bragg and R. Kleeman, On the alpha particles of radium and their loss of range in passing through various atoms and molecules. Philos. Mag. 10, 318-340 (1905).

43. D. Pines and $\mathrm{Ph}$. Nozières, The Theory of Quantum Liquids, Vol. 1, Normal Fermi Liquids. Addison-Wesley, Reading, PA, 1989.

44. D. Emfietzoglou, I. Abril, R. Garcia-Molina, I. D. Petsalakis, H. Nikjoo, I. Kyriakou and A. Pathak, Semi-empirical dielectric descriptions of the Bethe surface of the valence bands of condensed water. Nucl. Instrum. Methods B 266, 1154-1161 (2008).

45. T. Inagaki, R. N. Hamm, E. T. Arakawa and L. R. Painter, Optical and dielectric properties of DNA in the extreme ultraviolet. J. Chem. Phys. 61, 4246-4250 (1974).

46. J. A. LaVerne and S. M. Pimblott, Electron energy-loss distributions in solid, dry DNA. Radiat. Res. 141, 208-215 (1995).

47. N. D. Mermin, Lindhard dielectric function in the relaxationtime approximation. Phys. Rev. B 1, 2362-2363 (1970).

48. B. L. Henke, E. M. Gullikson and J. C. Davis, At. Data Nucl. Data Tables 54, 181 (1993). The ASCII files for the $f_{1}$ and $f_{2}$ scattering factors of the different elements can be downloaded from http://henke.lbl.gov/optical_constants/asf.html.

49. G. P. Williams, Electron binding energies, Sect. 1.1 in X-ray Data Booklet. Available at http://xdb.lbl.gov/Section1/Sec_1-1.html.

50. D. J. Planes, R. Garcia-Molina, I. Abril and N. R. Arista, Wavenumber dependence of the energy loss function of graphite and aluminum. J. Electron Spectrosc. Relat. Phenom. 82, 23-29 (1996).
51. C. D. Denton, I. Abril, R. Garcia-Molina, J. C. Moreno-Marìn and S. Heredia-Avalos, Influence of the description of the target energy-loss function on the energy loss of swift projectiles. Surf. Interface Anal. 40, 1481-1487 (2008).

52. S. Tanuma, C. J. Powell and D. R. Penn, Use of sum rules on the energy-loss function for the evaluation of experimental optical data. J. Electron Spectr. Related Phen. 62, 95-109 (1993).

53. P. Andreo, On the clinical spatial resolution achievable with protons and heavier charged particle radiotherapy beams. Phys. Med. Biol. 54, N205-N215 (2009).

54. M. Scholz and G. Kraft, Track structure and the calculation of biological effects of heavy charged particles. Adv. Space Res. 18, 5-14 (1996).

55. ICRU, Stopping Powers and Ranges for Protons and Alpha Particles. Report 49, International Commission on Radiation Units and Measurements, Bethesda, MD, 1993.

56. W. Brandt and M. Kitagawa, Effective stopping-power charges of swift ions in condensed matter. Phys. Rev. B 25, 5631-5637 (1982).

57. Ph. Bernhardt and H. G. Paretzke, Calculation of electron impact ionization cross sections of DNA using the Deutsch-Märk and Binary-Encounter-Bethe formalisms. Int. J. Mass Spectrosc. 223-224, 599-611 (2003).

58. P. Możejko and L. Sanche, Cross sections for electron scattering from selected components of DNA and RNA. Radiat. Phys. Chem. 73, 77-84 (2005).

59. M. A. Bernal and J. A. Liendo, The HKS model for electron production in liquid water by light ions. Nucl. Instrum. Methods B 251, 171-176 (2006).

60. L. Sanche, Low energy electron-driven damage in biomolecules. Eur. Phys. J. D 35, 367-390 (2005). 